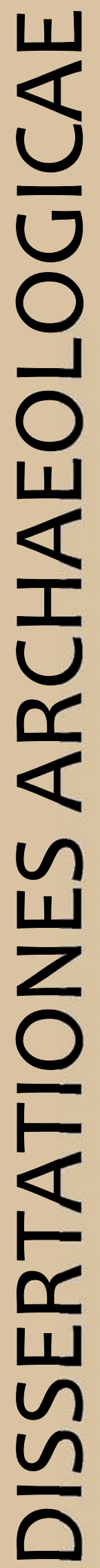

ex Instituto Archaeologico Universitatis de Rolando Eötvös nominatae

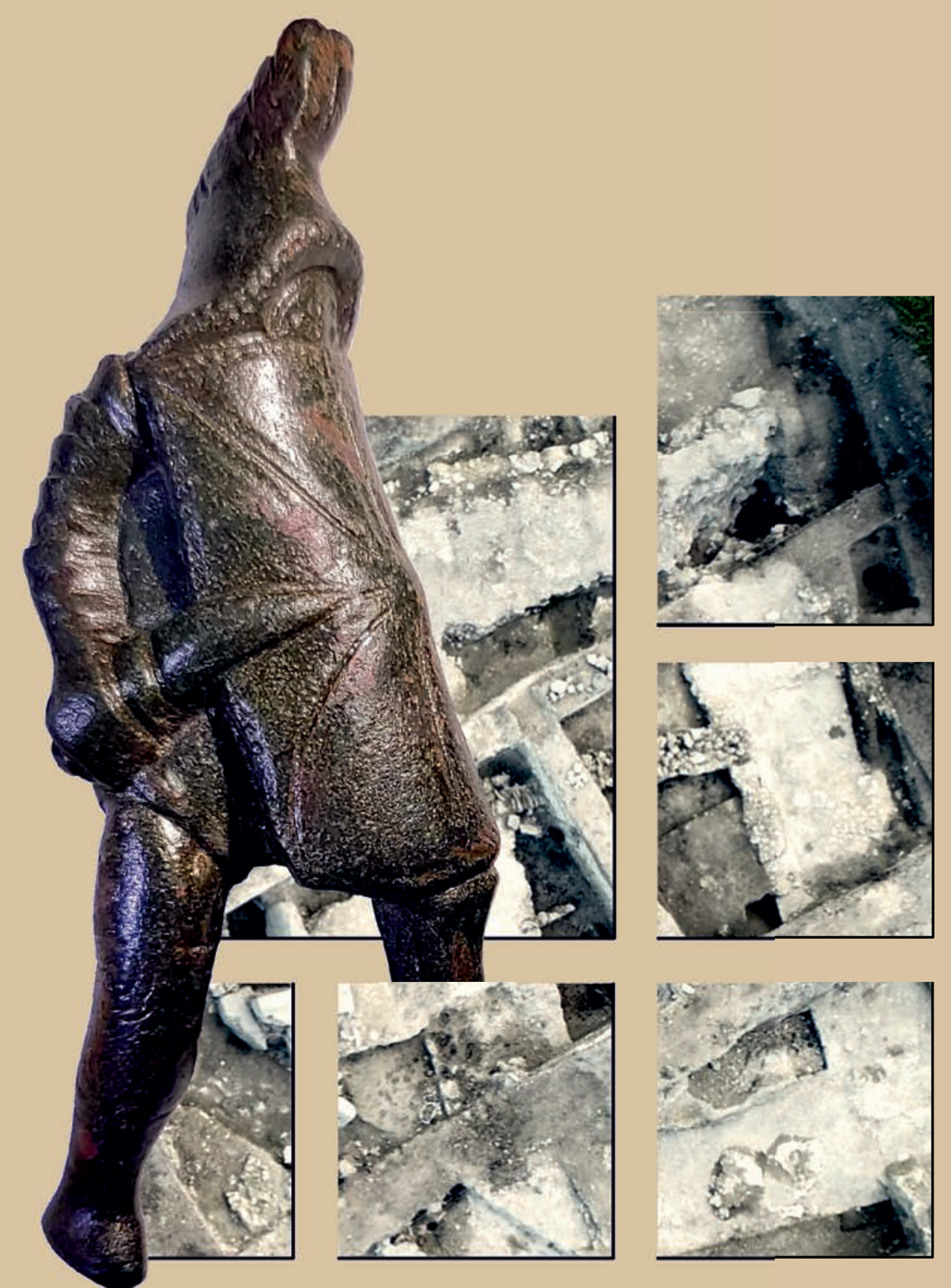

$$
\text { Ser. 3. No. 7. } 2019
$$




\section{Dissertationes Archaeologicae ex Instituto Archaeologico}

Universitatis de Rolando Eötvös nominatae Ser. 3. No. 7.

Budapest 2019 
Dissertationes Archaeologicae ex Instituto Archaeologico Universitatis de Rolando Eötvös nominatae

Ser. 3. No. 7.

Editor-in-chief:

DÁvid BARTUS

Editorial board:

LÁsZló BARTOSIEWICZ

LÁSZLÓ BORHY

ZOLTÁN CZAJLIK

IsTVÁN FELD

GÁBOR KALLA

PÁL RACZKY

MikLÓs SzABÓ

TivadAR VidA

Technical editor:

GÁBOR VÁCZI

Proofreading:

SZILVIA BARTUS-SZÖLLősI

ZsóFIA KondÉ

Aviable online at http://dissarch.elte.hu

Contact: dissarch@btk.elte.hu

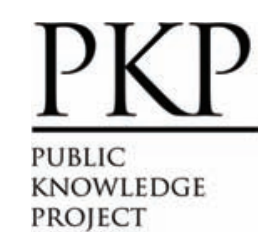

๑ ELTE Eötvös Loránd University, Institute of Archaeological Sciences

Layout and cover design: Gábor Váczi

Budapest 2019 


\section{CONTENTs}

\section{ARticles}

János Gábor TARBAY

The Casting Mould and the Wetland Find - New Data on the Late Bronze Age

Peschiera Daggers

Máté MeRvel

Late Bronze Age stamp-seals with negative impressions of seeds from Eastern Hungary

János Gábor TARBAY

Melted Swords and Broken Metal Vessels - A Late Bronze Age Assemblage

from Tatabánya-Bánhida and the Selection of Melted Bronzes

Ágnes ScHNEIDER

Multivariate Statistical Analysis of Archaeological Contexts: the case study

of the Early La Tène Cemetery of Szentlörinc, Hungary

Csilla SÁRÓ - Gábor LASSÁNYI

Bow-tie shaped fibulae from the cemetery of Budapest/Aquincum-Graphisoft Park

Dávid BARTus

Roman bronze gladiators - A new figurine of a murmillo from Brigetio

Kata DÉvAI

Re-Used Glass Fragments from Intercisa

Bence Simon

Rural Society, Agriculture and Settlement Territory in the Roman, Medieval and Modern Period Pilis Landscape

Rita RAKONCZAY

„Habaner“ Ofenkacheln auf der Burg Čabrad”

\section{FIELD REPORT}

Bence Simon - Anita Benes - Szilvia Joháczi - Ferenc BARnA

New excavation of the Roman Age settlement at Budapest dist. XVII, Péceli út (15127) site 


\section{Thesis Abstracts}

Kata SzILÁGYi

Die Silexproduktion im Kontext der Südosttransdanubischen Gruppe

der spätneolithischen Lengyel-Kultur

Norbert FARAGÓ

Complex, household-based analysis of the stone tools of Polgár-Csőszhalom

János Gábor TARBAY

Type Gyermely Hoards and Their Dating - A Supplemented Thesis Abstract

Zoltán Havas

The brick architecture of the governor's palace in Aquincum

Szabina Merva

'...circa Danubium...' from the Late Avar Age until the Early Árpádian Age-

$8^{\text {th }}-11^{\text {th }}$-Century Settlements in the Region of the Central Part of the Hungarian

Little Plain and the Danube Bend

Szabolcs Balázs NAGY

Noble Residences in the $15^{\text {th }}$ century Hungarian Kingdom - The Castles of Várpalota,

Ujlak and Kisnána in the Light of Architectural Prestige Representation

Ágnes KollátH

Tipology and Chronology of the early modern pottery in Buda 


\title{
Die Silexproduktion im Kontext \\ der Südosttransdanubischen Gruppe \\ der spätneolithischen Lengyel-Kultur
}

KATA SZILÁGYi

Móra Ferenc Museum, Szeged

szil.szvetlana@gmail.com

\begin{abstract}
Abstrakt
Dieser Artikel fasst eine Dissertation zusammen, die 2019 verteidigt wurde. Grundlage dieser Doktorarbeit bilden die fast 6200 Fundstücke von lithischem Material aus der Fundstelle Alsónyék-Bátaszék, Ungarn. Diese werden mit einer ähnlichen Menge an Silexmaterialien aus den Inventaren der anderen veröffentlichten spätneolithischen Fundstätten aus Südosttransdanubien verglichen. Als erstes Thema wird eine Charakterisierung der lokalen Verwendung von lithischen Rohstoffen in den verschiedenen lithischen Inventaren vorgestellt. Diese wurde im Zuge einer Prospektion untersucht, deren Ziel es war, die primären Rohstoffquellen zu lokalisieren und die prähistorischen Methoden der Rohstoffbeschaffung zu rekonstruieren. Das zweite Thema ist eine Analyse der Techniken zur Herstellung von Steinwerkzeugen und der Gebrauchsmuster von Artefakten. Auf dieser Grundlage wird ein Blick auf die gesamte chaîne opératoire der Steingeräteproduktion geworfen, wobei der Schwerpunkt auf dem Ablauf des Produktionsprozesses liegt, der von der Suche nach den Rohstoffquellen in ihrer spezifischen Umgebung über die Auswahl der Vorbereitungs- und Herstellungsverfahren innerhalb der Siedlung bis zur Deponierung in den Gräbern reicht. Dies gibt uns ein besseres Verständnis für die Bedeutung der natürlichen Umwelt, die Kenntnis des Rohstoffs und der Beschaffungsmethoden auf der Grundlage der Rohstoffauswahlstrategie der jeweiligen Gemeinschaften, in diesem Fall der südosttransdanubischen spätneolithischen Lengyel-Kultur. In der Siedlung lassen sich das technologische System der Gemeinschaft, mögliche Aktivitätszonen und Muster der Werkzeugnutzung durch das Studium der lithischen Artefakte rekonstruieren. Bei den in Bestattungen deponierten Steinwerkzeugen vollzieht sich eine Transformation von einem durchschnittlichen Gebrauchs-/Alltagssteinwerkzeug zu einem eindeutigen Symbol im Bestattungskontext, das wiederum auf die Umweltumgebung (,die physische Welt“) zurückgeführt werden kann.
\end{abstract}

\section{Die Ziele der Dissertation}

Die spätneolithische Lengyel-Kultur lässt sich auf dem Gebiet der heutigen Staaten Ungarn, Österreich, der Slowakei, der Tschechischen Republik, Polen, Slowenien und Kroatien lokalisieren. In Anbetracht der enormen geographischen Ausdehnung der Kultur wurden viele regionale Untergruppen durch die länderspezifische archäologische Forschung Ungarns und der Nachbarländer definiert. Die Lengyel-Kultur wurde nicht nur territorial, sondern auch chronologisch in mehrere Gruppen unterteilt, was zu zahlreichen terminologischen Schwierigkeiten für die Forschung führte. ${ }^{1}$

Die südost-transdanubische Gruppe kann als die „klassischste“ Einheit in der Forschung unseres Landes betrachtet werden, weil Mór Wosinsky die Lengyel-Kultur als eine unabhängige archäologische Einheit auf der Grundlage einer neolithischen Siedlung bestimmt hat, die an

1 RAcZKy 1974; Zalai-GaÁl 1980; BÁNFFy 1991; ZAlai-GaÁl 1993; BÁNFFy 1994; BÁNFFy 1995; RACZKY 1998; REgENYE 2000; RACZKY 2002; BÁNFFy 2007; REgENye 2011. 
der Grenze der Lengyel-Siedlung im Komitat Tolna zwischen 1882-1886 entdeckt wurde. Der nächste Meilenstein war die Ausgrabung von János Dombay in Zengővárkony, gefolgt von der Veröffentlichung des Platzes und des gesamten archäologischen Materials, die ein völlig neues Licht auf unser Bild der Kultur warfen. ${ }^{2}$ Später beschäftigte sich István Zalai-Gaál, dessen Arbeit sich insbesondere auf die Bestattungsaktivitäten dieser spätneolithischen Gemeinschaft konzentrierte, mit der südosttransdanubischen Gruppe der Lengyel-Kultur. ${ }^{3}$ Die von István Zalai-Gaál ausgegrabene Stätte Mórágy-Tűzkődomb stellte einen wichtigen Beitrag für die Erforschung dieser Kultur dar, da die dort gefundenen Hunderte von Gräbern nicht nur einen Durchbruch in der Quantität, sondern auch in der Qualität der Informationen darstellten. ${ }^{4}$ In den 2000er Jahren berührten die groß angelegten archäologischen Ausgrabungen im Zusammenhang mit dem Autobahnbau auch das Gebiet Südtransdanubiens, was zur Entdeckung der Stätte Alsónyék-Bátaszék führte. ${ }^{5}$ Die Ausgrabung wurde durch den Autobahnbau notwendig, der eine groß angelegte Oberflächenuntersuchung einleitete, die die Möglichkeit eröffnete, eine Fläche von 25 Hektar zu untersuchen, die eine der größten neolithischen Stätten in Mitteleuropa feilegte. ${ }^{6}$ Dank der großen Ausgrabungsfläche konnte eine große Anzahl von Gebäuden und Gräbern zu dem bisher Bekannten hinzugefügt werden. Dies bedeutet, dass wir nun in Bezug auf die Forschungsgeschichte der Lengyel-Kultur eine neue Dimension empirischen Wissens erschließen können (Abb. 1). ${ }^{7}$

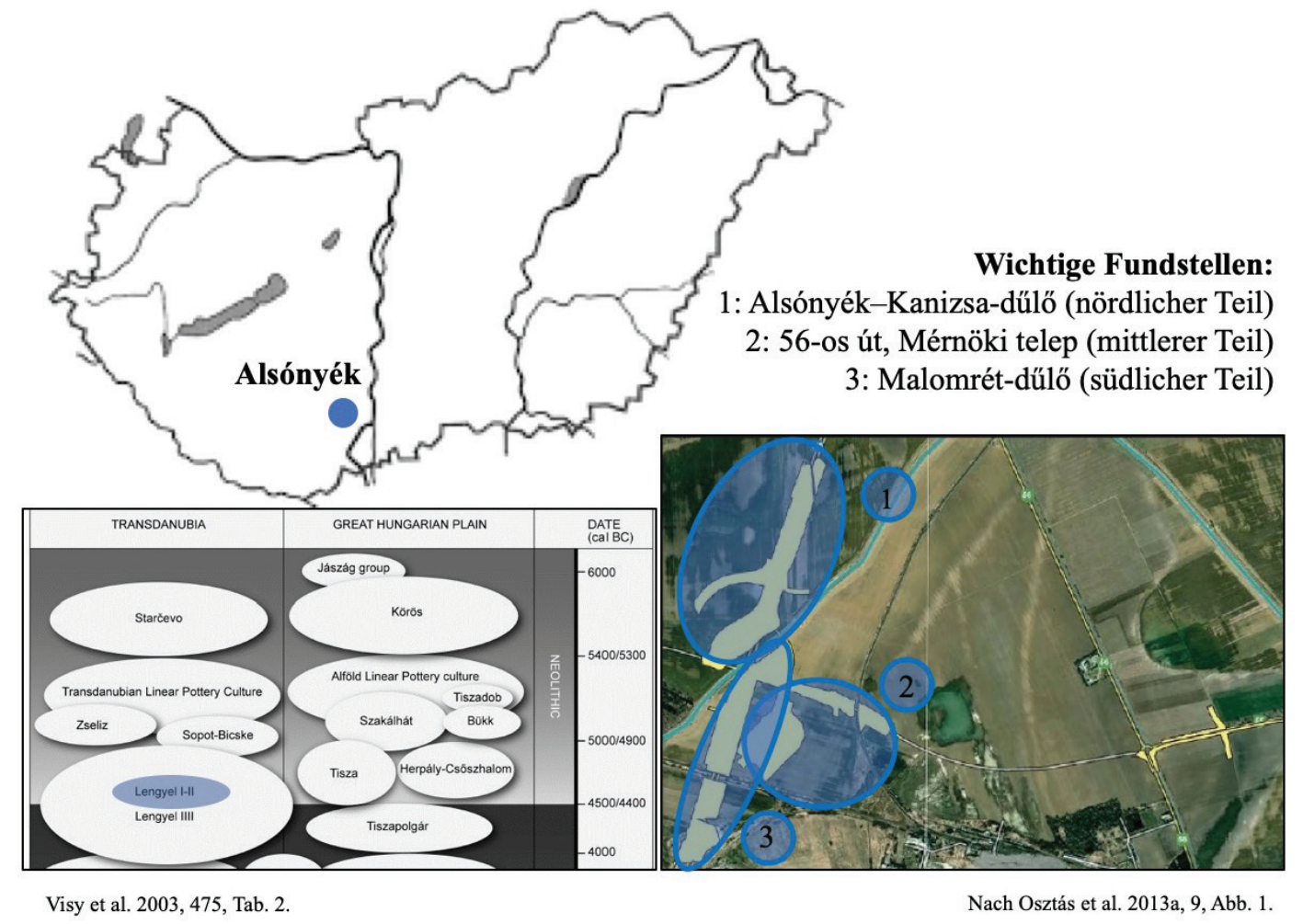

$A b b$. 1. Die Lage des Alsónyék und seiner Unterstandorte (Ausgrabungsflächen). Erstellt von Kata Szilágyi.

2 DOMBAY 1939; DOMBAY 1958; DOMBAY 1960.

3 ZALAI-GAÁl 1982; ZALAI-GAÁl 1986; ZALAI-GAÁl 1988.

4 ZalAi-GaÁl 2001; ZALAI-GAÁl 2002.

5 ZaLAI-GAÁL - OsZTÁs 2009, 245.

6 Osztás et al. 2012, 377-378; SERLEGI et al. 2013, 5.

7 OsztÁs et al. 2013a; Osztás et al. 2013b. 
Grundlage dieser Doktorarbeit sind die fast 6200 Fragmente von Silexmaterial aus dem Standort Alsónyék-Bátaszék. In der Dissertation beschränkt sich die Untersuchung der Steinwerkzeuge auf die Artefakte, die aus den Siedlungsobjekten und Gräbern der spätneolithischen Lengyel-Kultur stammen ( $A b b .2)$. Insbesondere das Studium des Prozesses der Herstellung von Silexwerkzeugen spielt eine entscheidende Rolle, wie es heute auch bei anderen Artefakttypen immer häufiger der Fall ist: Als geeignete Methode zur Rekonstruktion des Produktionsprozesses wird das Konzept der chaîne opératoire verwendet. ${ }^{8}$ Diese Konzept geht davon aus, dass sich bei der Herstellung von Steinwerkzeugen verschiedene Phasen der Rohstoffbeschaffung, der Produktion und der Nutzung unterscheiden lassen, gefolgt von der Entsorgung oder Deponierung - alle diese Phasen gehören zur Biographie der Artefakte. ${ }^{9}$ Die Grundstruktur der Dissertation spiegelt diesen Ansatz wider.

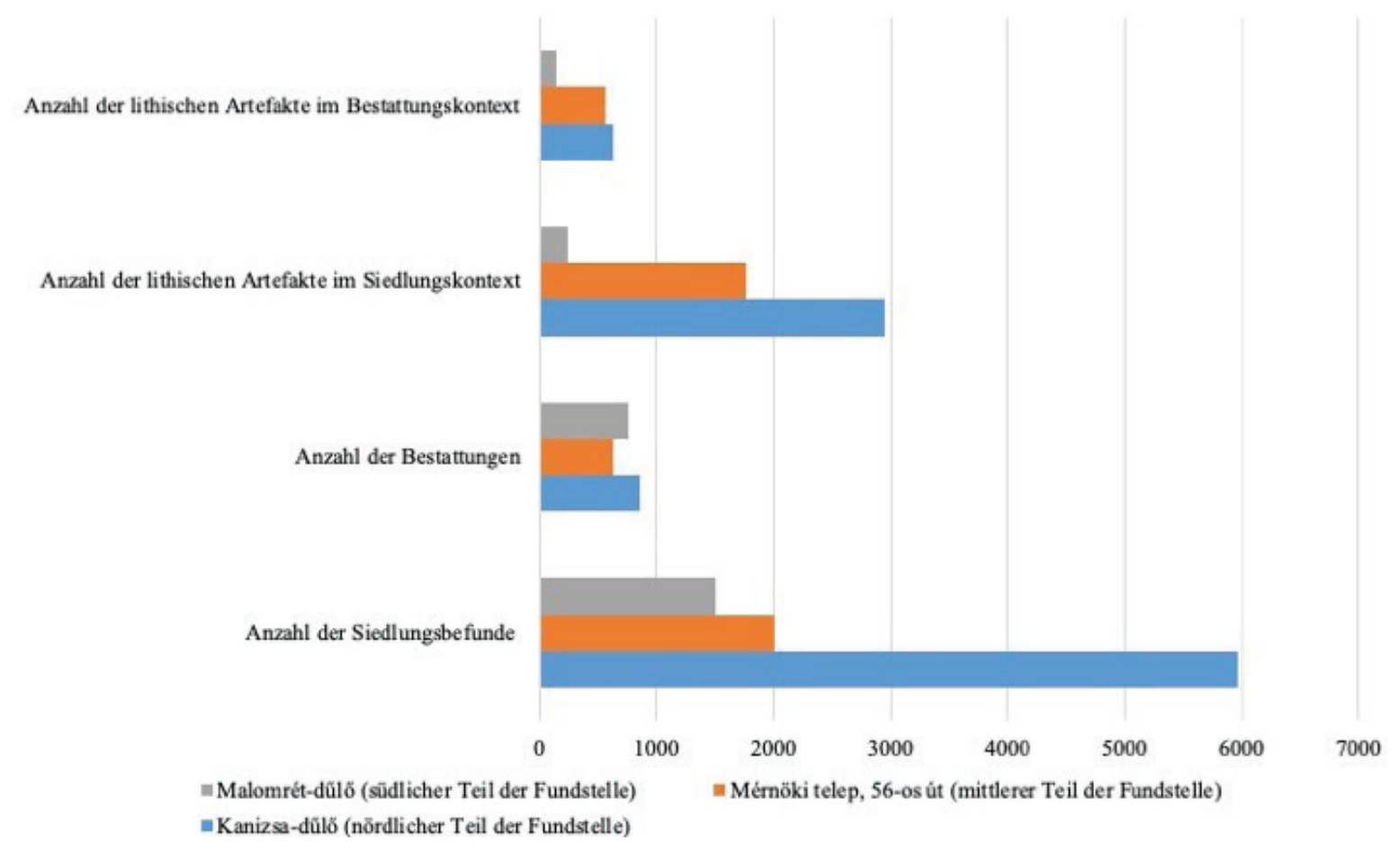

Abb. 2. Vergleich der Anzahl archäologischer Befunde und lithischer Artefakte.

Eine der Säulen meiner Forschung war die Klärung der Lage und Position von Rohstoffquellen, die mit einem geoarchäologischen Ansatz durchgeführt wurde..$^{10}$ Das primäre Ziel war es, das Spektrum des abbauwürdigen Rohstoffs auf dem Gebiet des Ost-Mecsek-Gebirges zu erfassen und zu dokumentieren. Außerdem wollte ich eine Sammlung der verfügbaren geologischen Proben erstellen, die einen Vergleich zwischen den archäologischen Steinwerkzeugen und geologischen Proben ermöglicht. Der Mecsek-Radiolarit dominiert im lithischen Material von Alsónyék, seine geologische Quelle liegt 30-50 km von der Fundstelle entfernt auf dem Gebiet des Ost-Mecsek (Abb. 3). Ich habe das Thema der Rohstoffbeschaffung durch eine Felduntersuchung genauer untersucht. Ziel war es, die Strategie der Rohstoffauswahl, die Sammel- und/oder Abbautätigkeit der Gemeinschaften des Spätneolithikums zu verstehen. ${ }^{11}$

8 InIZAN et al. 1999, 13-17; Odell 2006, 1-12; ANDREFSKy 2008, 3-13.

9 Holló et al. 2001; Holló et al. 2002; Holló et al. 2004; ANDrefsKy 2009, 66-70.

10 SzILÁgyi 2018a, 1-9.

11 SzILÁGYi 2018b, 130-132. 


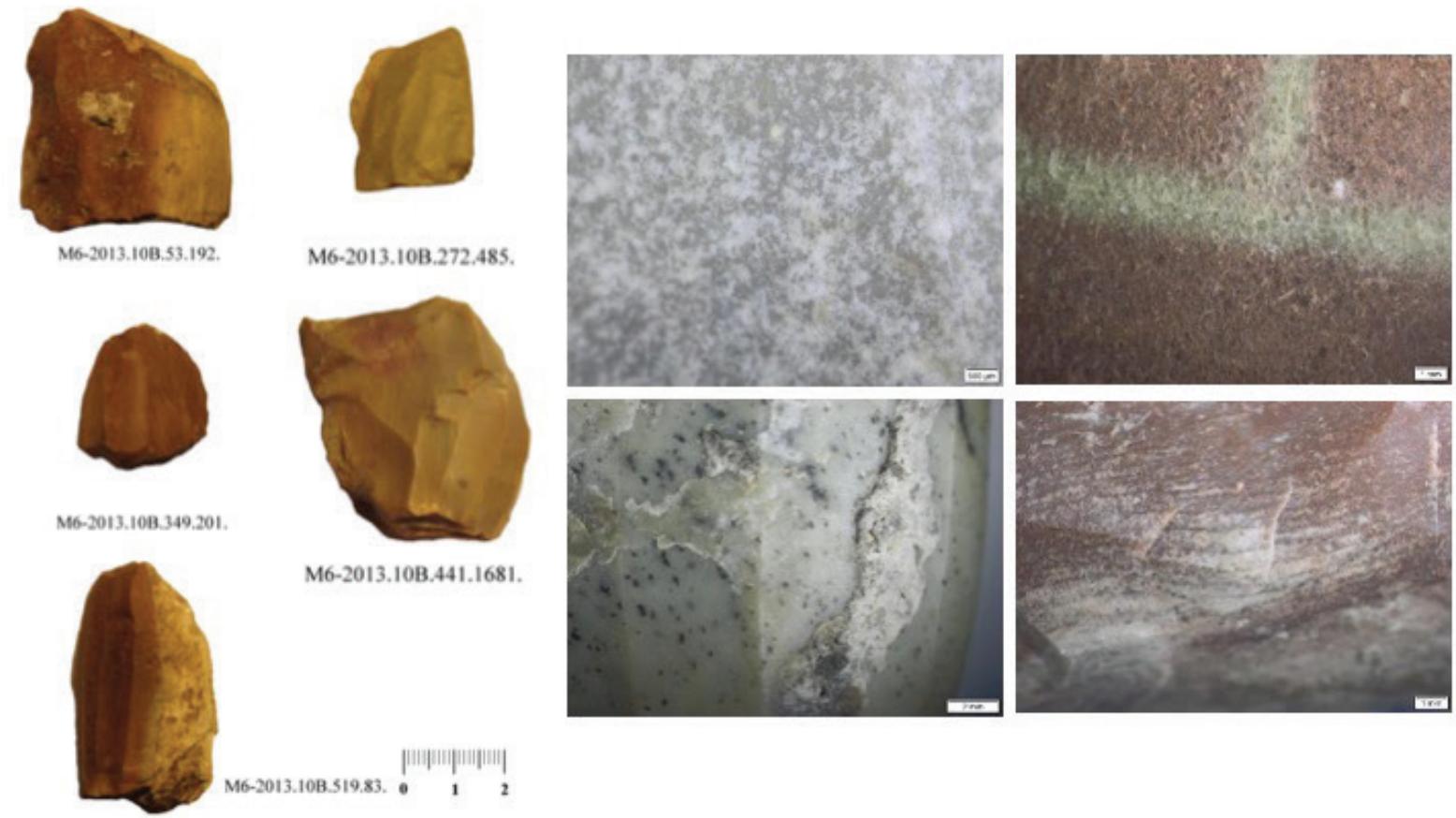

$A b b$. 3. Verschiedene Farb- und Mustervariationen von Mecsek radiolarit (Alsónyék-Bátaszék).

Als nächstes untersuche ich den gesamten Prozess der Herstellung von Steinwegzeugen, alle Phasen der Vorbereitung von Steinwerkzeugen und die Produktionstätigkeit, also den praktischen Prozess der Steinwerkzeugherstellung - dem chaîne opératoire-Ansatz entsprechend bestehend aus einzelnen, registrierbaren Gesten des Handwerkers. Zunächst wurden primäre analytische und vergleichende Einheiten unter den lithischen Materialien aus den Siedlungsobjekten und Gräbern definiert ( $A b b$. 4). Ich verglich die primären Merkmale - die Rohstoffauswahl, die technologischen Eigenschaften der Artefakte und ihre räumliche Verteilung - um einen umfassenden Überblick über das gesamte lithische Material zu erhalten. Mit dieser Methode ist es möglich, die Werkzeugherstellung innerhalb der Siedlung zu rekonstruieren, was die intensive Natur der Steingeräteproduktion in der spätneolithischen Siedlung offenbart. ${ }^{12}$

\begin{tabular}{|l|l|l|l|l|}
\hline \multicolumn{1}{|c|}{ Rohmaterial } & \multicolumn{1}{c|}{ Siedlung } & \multicolumn{1}{c|}{ Prozent (\%) } & \multicolumn{1}{c|}{ Bestattung } & \multicolumn{1}{c|}{ Prozent (\%) } \\
\hline Mecsek radiolarit & 3324 & 67,19 & 616 & 46,92 \\
\hline Bakony radiolarit & 812 & 16,41 & 405 & 30,85 \\
\hline Radiolarit & 328 & 6,63 & 113 & 8,61 \\
\hline Feuerstein & 194 & 3,92 & 46 & 3,50 \\
\hline Obsidian & 131 & 2,65 & 36 & 2,74 \\
\hline Limnosilices & 22 & 0,44 & 18 & 1,37 \\
\hline Unbekannt & 114 & 2,30 & 19 & 1,45 \\
\hline Entferntes Rohmaterial & 22 & 0,44 & 60 & 4,57 \\
\hline Gesamt & 4947 & 100,00 & 1313 & 100,00 \\
\hline
\end{tabular}

Tab. 1. Die Anzahl und der prozentuale Anteil des Rohmaterials in den Siedlungs- und Begräbnissammlungen von Alsónyék-Bátaszék. 


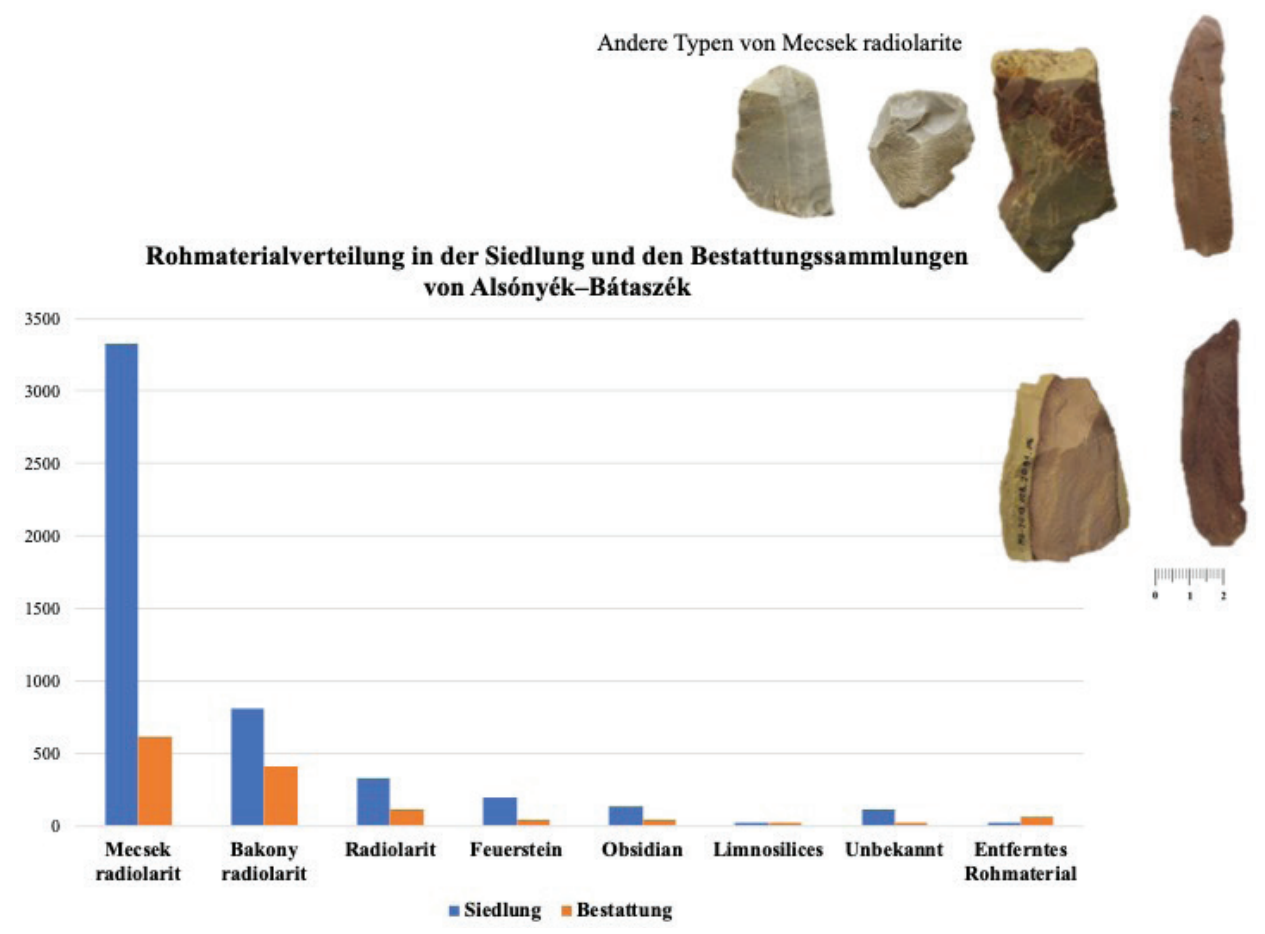

Abb. 4. Rohmaterialverteilung in der Siedlung und den Bestattungssammlungen von Alsónyék-Bátaszék.

Die Annäherung an kleinere Analyseeinheiten der lithischen Materialien der Siedlung und der Gräber erforderte eine andere methodische Herangehensweise: Hier konzentrierte sich die Untersuchung auf die quantitative räumliche Verteilung der Artefakte in Bezug auf die archäologischen Befunde, die mit den Gebäuden in der Siedlung verbunden werden können. Hier berühre ich das Feld der Haushaltsarchäologie, und der grundlegende Punkt war es, diejenigen Gruben zu identifizieren und besser zu verstehen, die zu einem oder mehreren Häusern gehörten, die eine Haushaltseinheit bildeten. ${ }^{13}$ Die Frage, wo und wie die Werkzeugherstellung erfolgte ist eine komplexe Frage, die die Forschung und die Verarbeitung und Interpretation der lithischen Assemblage aus der Siedlung leitete (Abb. 5).

\begin{tabular}{|c|c|c|c|c|c|c|c|c|c|}
\hline $\begin{array}{c}\text { Technologische } \\
\text { Kategorie/ } \\
\text { Rohmaterial }\end{array}$ & 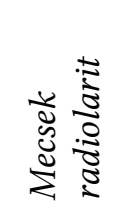 & 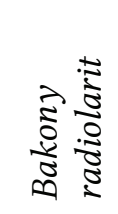 & 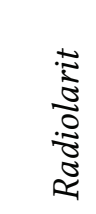 & 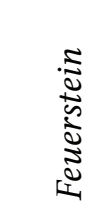 & $\begin{array}{l}\vec{\Xi} \\
\tilde{J} \\
\frac{\tilde{J}}{\tilde{J}} \\
\frac{\tilde{J}}{5}\end{array}$ & 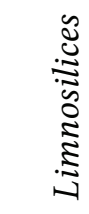 & $\begin{array}{l}\tilde{5} \\
: \overrightarrow{0} \\
\tilde{5} \\
\tilde{0}\end{array}$ & 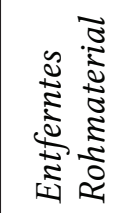 & 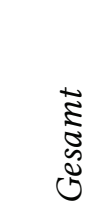 \\
\hline Kern & 483 & 104 & 27 & 13 & 2 & 2 & 8 & 2 & 641 \\
\hline Rohmaterial-Block & 102 & 21 & 14 & 18 & 77 & 6 & 0 & 0 & 238 \\
\hline Abschlag & 627 & 224 & 78 & 42 & 10 & 2 & 44 & 4 & 1031 \\
\hline Klinge & 1331 & 373 & 159 & 95 & 21 & 10 & 78 & 9 & 2076 \\
\hline Werkzeug & 781 & 90 & 50 & 26 & 4 & 2 & 1 & 7 & 961 \\
\hline Gesamt & 3324 & 812 & 328 & 194 & 114 & 22 & 131 & 22 & 4947 \\
\hline
\end{tabular}

Tab. 2. Technologische Kategorie und Rohmaterialverteilung im Siedlungskontext von AlsónyékBátaszék.

13 SzILÁgyi 2017a. 
Technologische Kategorie und Rohmaterialverteilung im Siedlungskontext

$(n=4947)$

2500

2000

1500

1000

500
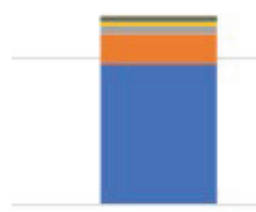

Kern

Rohmaterial-Block

- Mecsek radiolarit

Unbekannt
Eakony radiolarit

- Limnosilices

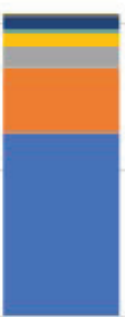

Abschlag

= Radiolarit

- Obsidian

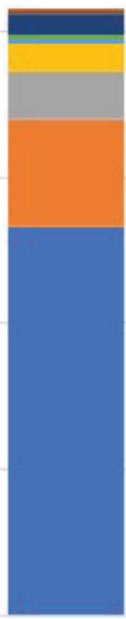

\section{Klinge}

Feuerstein

- Entferntes Rohmaterial

Werkzeug

Abb. 5. Die Rohmaterialverteilung in den Siedlungskontexten.

Technologische Kategorie und Rohmaterialverteilung im Bestattungskontext $(\mathrm{n}=1313)$

1000
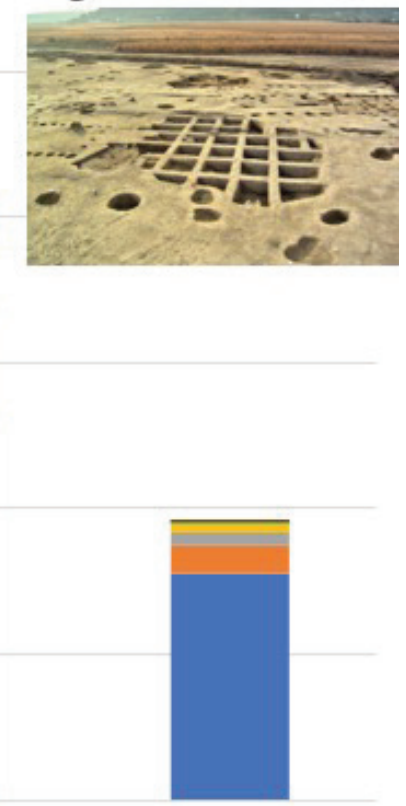
Da die Steinwerkzeuge, die als Grabbeigaben deponiert wurden, eine absichtlich ausgewählte Probe darstellen, die von Werte- und Bedeutungssystemen beherrscht wird, müssen wir diese Inventare mit einer anderen Methode erforschen und interpretieren als die aus den Siedlungen. Am Fundort Alsónyék gehörte der Hauptteil der 2236 ausgegrabenen Gräber zu deutlich sichtbaren Grabgruppen. Dies repräsentiert eine Transformation der sozialen und kognitiven Systeme während des Spätneolithikums, denn in der vorangegangenen Periode lagen die Gräber zufällig und sporadisch zwischen Häusern und verschiedenen Orten in der Siedlung. ${ }^{14}$ Wir können die Grabgruppen in Alsónyék als Muster einer konvergierenden Bestattungspraxis verstehen, da wir im Vergleich mit der vorhergehenden Periode ein regelmäßigeres Muster in der Ausrichtung der Gräber, der Position der bestatteten Personen und den Merkmalen der Grabbeigaben erfassen. Vor diesem Hintergrund stellt sich vor allem die Frage, wie die lithischen Materialien als Grabbeigabe von Bedeutung sind und was sie im Bestattungskontext bedeuten. Das Rohmaterial, der Artefakttyp und die genaue Position sind konkrete Zeichen, die Symbole jenseits der reinen Materialität der Artefakte darstellen. Ich habe versucht, die möglichen Deutungen durch Kontextualisierung der verfügbaren anthropologischen Daten aufzudecken und nach Korrelationen von Objektmustern in Bezug auf das biologische Geschlecht und das Sterbealter zu suchen (Abb. 6). ${ }^{15}$

\begin{tabular}{|c|c|c|c|c|c|c|c|c|c|}
\hline $\begin{array}{l}\text { Technologische } \\
\text { Kategorie/ } \\
\text { Rohmaterial }\end{array}$ & 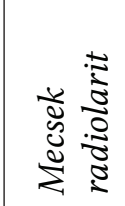 & 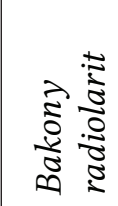 & 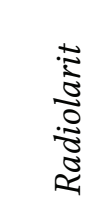 & 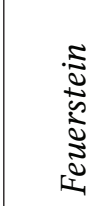 & $\begin{array}{l}\text { है } \\
\tilde{\Xi} \\
\text { Jँ } \\
\tilde{\Xi} \\
\text { है }\end{array}$ & 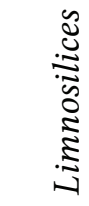 & $\begin{array}{l}\tilde{5} \\
: \tilde{\Xi} \\
\tilde{0} \\
0\end{array}$ & 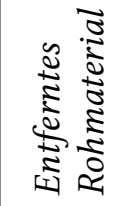 & 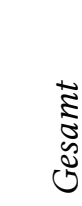 \\
\hline Kern & 21 & 6 & 1 & 1 & 0 & 0 & 6 & 1 & 36 \\
\hline $\begin{array}{l}\text { Rohmaterial- } \\
\text { Block }\end{array}$ & 11 & 2 & 0 & 10 & 14 & 0 & 0 & 0 & 37 \\
\hline Abschlag & 40 & 27 & 13 & 3 & 0 & 0 & 5 & 0 & 88 \\
\hline Klinge & 402 & 275 & 76 & 29 & 3 & 0 & 25 & 57 & 867 \\
\hline Werkzeug & 142 & 95 & 23 & 3 & 2 & 18 & 0 & 2 & 285 \\
\hline Gesamt & 616 & 405 & 113 & 46 & 19 & 18 & 36 & 60 & 1313 \\
\hline
\end{tabular}

Tab. 3. Technologische Kategorie und Rohmaterialverteilung im Bestattungskontext von AlsónyékBátaszék.

Schließlich verglich ich in einer breiteren Perspektive das Silexmaterial von Alsónyék mit den veröffentlichten und unveröffentlichten lithischen Inventaren der südöstlich-transdanubischen Gruppe der Lengyel-Kultur (Zengővárkony, Mórágy-Tűzkődomb, Pécsvárad-Aranyhegy, Lengyel-Sánc, Villánykövesd). ${ }^{16}$ Mein Ziel war es, die Tätigkeit der spätneolithischen Gemeinschaften im Bereich der Herstellung von Steinwerkzeugen zu verstehen und zu interpretieren, da die lithischen Inventare der genannten Fundorte in Bezug auf Rohstoffe und technologische Kategorien sehr ähnlich sind. Aus diesem Grund möchte ich die Strategien der Werkzeugherstellung, die technologischen Gesamtsysteme und den Wert und die Bedeutung des lokalen Rohstoffs in der breiteren kulturellen Einheit ,Lengyel' untersuchen.

14 Zalai-GaÁl et al. 2012a; Zalai-GAÁl et al. 2012b.

15 KÖHLER 2012; KÖHLER 2013.

16 BÁcskay - T. Biró 1984; BÁcskay 1989; T. Biró 1989; BÁcsKay 1990; T. Biró 1990. 
Zusammenfassend lassen sich die Themen der Dissertation in drei grundlegenden, aber insgesamt sehr komplexen Fragen zusammenfassen:

- 1. Welche Arten von Werkzeugherstellungsverfahren und Produktionsaktivitäten wurden speziell in Alsónyék und in der südost-transdanubischen Gruppe der Lengyel-Kultur eingesetzt?

- 2. Welche Bedeutung und welchen symbolischen Wert hatten - insbesondere lokale Rohstoffe im Leben dieser spätneolithischen Gemeinschaft?

- 3. Wie können wir die neuen Bestattungspraktiken verstehen, die in Alsónyék beobachtet wurden? Was symbolisierten der Niederlegungsort, das Rohmaterial und die technologisch/typologischen Merkmale der Steinwerkzeuge im Bestattungskontext?

\section{Methoden und Quellen}

Seit den 1970er Jahren hat der technologische Ansatz (chaîne opératoire) in der ungarischen prähistorischen Forschung immer mehr an Bedeutung gewonnen, was sich besonders bei der Erforschung von Silexartefakten bemerkbar macht. In der französischen prähistorische Forschung hat der technologische Ansatz hat eine lange Tradition und er spielt eine wichtige Rolle, was in sehr genauen terminologischen Definitionen und einer systematischen Auseinandersetzung mit Herstellungsverfahren von silexartefakten resultiert. ${ }^{17}$ Die technologische Sichtweise und das Bearbeitungsverfahren kristallisierten sich vor allem mit dem Schwerpunkt auf paläolithische Steinmaterialien heraus. Der Gesamterfolg dieser Methode zeigt jedoch deutlich, dass sie allgemein anwendbar und daher heute in fast allen archäologischen Epochen weit verbreitet ist. Die technologische Herangehensweise an die Untersuchung der verschiedenen Epochen und Artefakttypen ermöglichte kontinuierliche methodische Entwicklungen, die zu einer neuen Perspektive nicht nur auf neue Materialien, sondern auch auf die bereits publizierten Fundmaterialien führten. Dies kann eine neue Diskussion anregen, die die Möglichkeit bietet, diese neuen Erkenntnisse auf neue Weise zu sehen.

Die kürzlich veröffentlichte Fundstelle von Alsónyék bietet die Möglichkeit, mit diesem technologischen Ansatz völlig neue Erkenntnisse zu gewinnen, nicht nur in Bezug auf die südosttransdanubische Gruppe der Lengyel-Kultur, sondern auch in Bezug auf das gesamte Spätneolithikum. Dies ist der Fall, weil die Zahl der geschlagenen Steinwerkzeuge und die archäologischen Befunde in Alsónyék die bisher aus allen anderen Stätten der Lengyel-Kultur bekannten bei weitem übertreffen. Allein in Alsónyék wurden mehr Steinwerkzeuge gefunden als in allen anderen veröffentlichten lithischen Inventare aus Südost-Transdanubien zusammen. Da der archäologische Kontext in Alsónyék bekannt ist, bietet dies eine grundlegend verbesserte Möglichkeit, die spätneolithischen Silexherstellungsaktivitäten in ein neues Licht zu rücken.

Die meisten der bisher bekannten lithischen Inventaren wurden von Erzsébet Bácskay und Katalin T. Biró untersucht und veröffentlicht, aus deren Werken wir viele Details über die Rohstoffbeschaffung und die Produktionstätigkeit dieser spätneolithischen Gemeinschaften kennen. ${ }^{18}$ Auf dieser Grundlage wissen wir, dass der lokale Mecsek-Radiolarit der wichtigste Rohstoff war. Doch obwohl dieser Radiolarittyp räumlich nahe verfügbar ist, wurden auch

17 INIZAN et al. 1999.

18 BÁcskay - T. Biró 1984; BÁcskay 1989; T. Biró 1989; BÁcskAY 1990; T. Biró 1990. 
andere Radiolarit- und Silexarten verwendet. ${ }^{19}$ Klingen und retuschierte Werkzeuge auf Klingen dominieren die lithischen Inventare, darauf aufbauend wurde die Lengyel-Kultur auch als „Klingen-Kultur“ bezeichnet (Abb. 7).

\begin{tabular}{|l|l|l|l|l|l|l|}
\hline \multicolumn{1}{|c|}{$\begin{array}{c}\text { Rohmaterial/ } \\
\text { Fundstellen }\end{array}$} & Zengővárkony & Pécsvárad & Villánykövesd & Lengyel & Mórágy & Gesamt \\
\hline Mecsek radiolarit & 2530 & 462 & 53 & 386 & 191 & 3622 \\
\hline Bakony radiolarit & 20 & 6 & 4 & 49 & 13 & 92 \\
\hline Limnosilices & 3 & 1 & 1 & 8 & 0 & 13 \\
\hline Obsidian & 158 & 2 & 13 & 55 & 13 & 241 \\
\hline $\begin{array}{l}\text { Nordischer } \\
\text { Feuerstein }\end{array}$ & 8 & 0 & 4 & 1 & 0 & 13 \\
\hline $\begin{array}{l}\text { Andere } \\
\text { Rohmaterialien }\end{array}$ & 124 & 12 & 3 & 101 & 8 & 248 \\
\hline Gesamt & 2843 & 483 & 78 & 600 & 225 & 4229 \\
\hline
\end{tabular}

Tab. 4. Rohmaterialverteilung in den verglichenen Fundstellen der Lengyel-Kultur in Südost-Transdanubien.

Die lithische Assemblage von Alsónyék bietet eine ausgezeichnete Gelegenheit, den gesamten Werkzeugbau der südosttransdanubischen Gruppe der Lengyel-Kultur aus einer neuen Perspektive zu überdenken und zu verstehen, da unsere genaue Kenntnis der archäologischen Zusammenhänge in Alsónyék eine viel detailliertere Kontextanalyse ermöglicht.

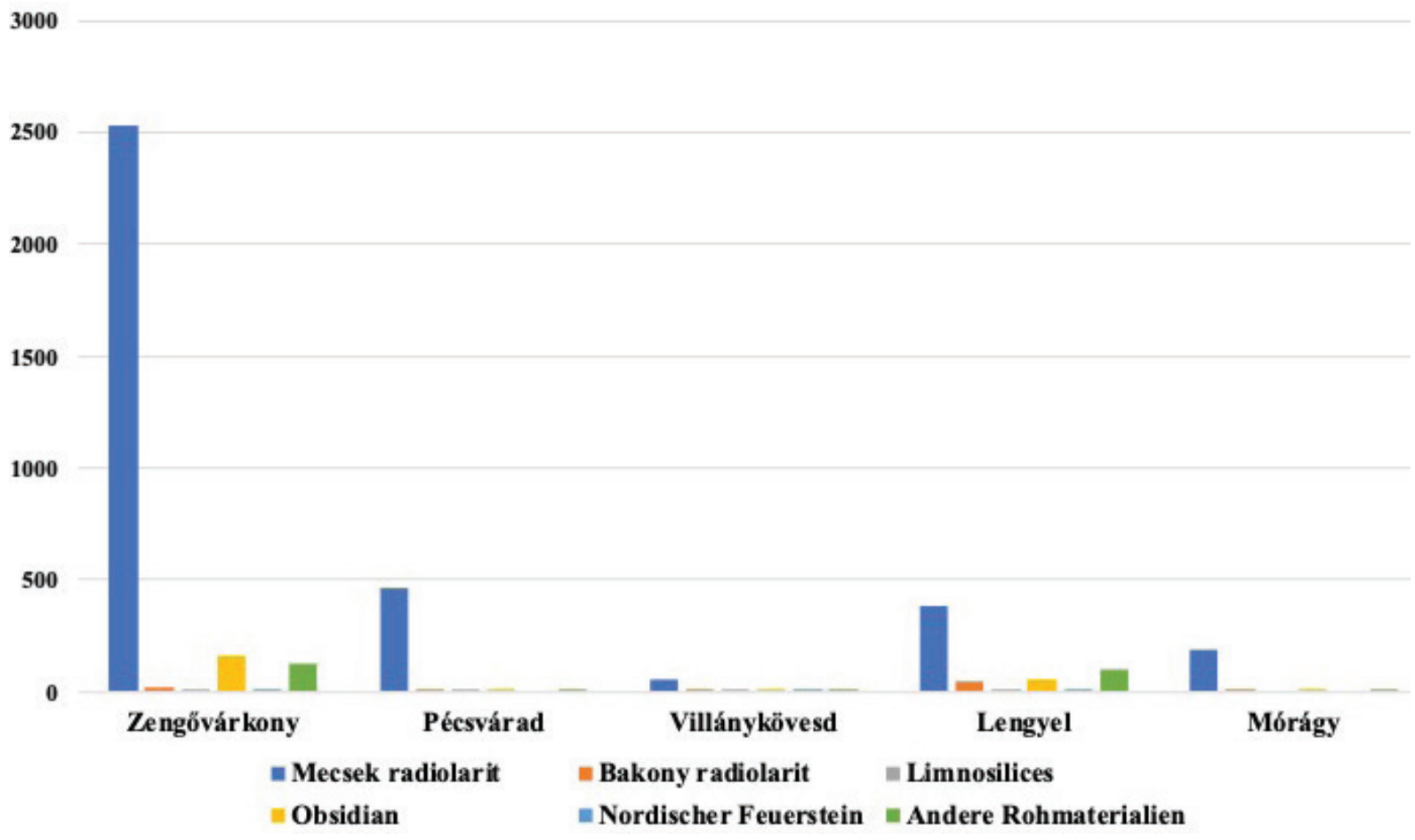

$A b b$. 7. Rohmaterialverteilung in den verglichenen Fundstellen der Lengyel-Kultur in Südost-Transdanubien $(n=4229)$.

19 T. Biró 1984; T. Biró 1988; T. Biró 1998. 
Der Vergleich der lithischen Inventare aus der Siedlung und den Gräbern bildet den Kern der Dissertation und wird im Kontext der größeren südost-transdanubischen Gruppe interpretiert. Ich interpretiere das gesamte lithische Material als Ausdruck einer grundlegenden Transformation im Spätneolithikum, die in den einzigartigen Merkmalen der Fundstelle sichtbar ist und keine Ähnlichkeiten mit den früheren mittelneolithischen und nachfolgenden frühkupferzeitlichen Fundstätten in Transdanubien aufweist. ${ }^{20}$ Aus diesem Grund konzentriere ich mich darauf, die Art dieser Transformation und die zugrunde liegenden Ursachen zu untersuchen und für eine ernsthafte soziale und mentale Transformation zu plädieren. Die gesamte Stätte - mit ihrer enormen Ausdehnung und der übermäßigen Anzahl von Gebäuden und Gräbern, mit ihrem einzigartigen archäologischen Material - hebt sich nicht nur von den anderen südosttransdanubischen Fundstätten des Spätneolithikums ab, sondern stellt auch einen Ausnahmefall im gesamten Gebiet Transdanubiens dar. Die Chronologie der Stätte umfasst angesichts der sehr intensiven Konzentration von Siedlungsstrukturen und Bestattungsaktivitäten einen überraschend kurzen Zeitraum: Auf der Grundlage der höchsten Wahrscheinlichkeit der verfügbaren ${ }^{14} \mathrm{C}$-Daten wurde die Siedlung 200-500 Jahre lang genutzt, während die Bestattungsaktivität 250-350 Jahre umfasst. Noch kürzer war dieser Zeitraum im am intensivsten genutzten Teil der Siedlung, wo die Lebensdauer mit 95 prozentiger Wahrscheinlichkeit auf 45 Jahre geschätzt wird, während die Bestattungsaktivitäten etwa 95 Jahre dauerten. ${ }^{21}$

In Bezug auf die gesamte Fundstelle untersuche ich die wichtige historische Frage, wie der Ursprung und die kurze Dauer dieses spätneolithischen Phänomens zu verstehen und zu interpretieren ist, und wie die außerordentlich intensive und konzentrierte Tätigkeit für eine begrenzte Anzahl von Generationen zu verstehen ist. Aus dieser Beobachtung können wir eine relativ „plötzliche“ Transformation ableiten, ein rasches Bevölkerungswachstum, das die Errichtung der ausgedehnten Siedlung erforderlich machte, Veränderungen in der Nahrungsmittelproduktion und neue Entwicklung handwerklicher Tätigkeiten. Um das Alsónyék-Phänomen“ zu verstehen, müssen wir die möglichen räumlichen und zeitlichen Dimensionen der Transformation untersuchen. Hinsichtlich der räumlichen Dimension unterscheidet sich Alsónyék von den anderen bekannten Stätten der Lengyel-Kultur insofern, als dass Alsónyék eine außerordentlich ausgedehnte Flachsiedlung ist. Was die zeitliche Dimension betrifft, so zeigt diese Periode eine außerordentliche Veränderungsdynamik, einen ausgeprägten sozialen und mentalen Wandel am Ende des Neolithikums und zu Beginn der Kupferzeit. Diese dynamische Veränderung wird durch die Grabbeigaben und Bestattungspraktiken sehr gut repräsentiert. Sie können in Bezug auf die größere regionale Transformation interpretiert werden, die in der benachbarten Großen Ungarischen Tiefebene sichtbar ist. In diesem Gebiet wurden zu dieser Zeit die Tell-Siedlungen nicht mehr genutzt, und es entstanden neue Gemeinschaften mit völlig anderer materieller Kultur und neuen Siedlungsformen. Anstelle von intramuralen Bestattungen (innerhalb der Siedlung) werden die Toten nun auf extramuralen Friedhöfen (außerhalb der Siedlung) begraben..$^{22}$ In Transdanubien war die Übergangsperiode zwischen diesen beiden Perioden (dem Spätneolithikum und der frühen Kupferzeit) von kontinuierlicher Natur, und die neue Kupferzeitperiode begann hier im Kontext derselben Lengyel-Kultur. In dieser Periode war die „Alsónyék“-Gemeinschaft wahrscheinlich der

20 BÁNfFy 1991, 23-33; BÁNFFy et al. 2013, BÁNFFy et al. 2014.

21 OszTÁs et al. 2013b, 280. Tab. 6, 282. Tab. 8.

22 RACZKy - ANDERs 2009; Siklósi 2010; SAlisbury 2012; Siklósi 2013; Raczky - Anders 2014; Raczky et al. 2014, 328-332; SCHIER 2014, 428. 
Agent dieser Transformation, während das Keramikmaterial darauf hindeutet, dass sie gleichzeitig ein Empfänger südlicher Impulse war. ${ }^{23}$

Aus diesem Grund konzentriere ich mich in der Dissertation auf eine weitere Untersuchung dieser Transformation. Dazu untersuche ich drei Hauptthemen, nämlich den Umwelthintergrund, die Aktivitäten in der Siedlung und die mit den Bestattungen verbundenen Aktivitäten. Innerhalb jedes dieser Themen werden verschiedene Phänomene auf unterschiedlichen räumlichen Skalen und mit unterschiedlichen Methoden untersucht. Während bei der Untersuchung des Umwelthintergrunds die Wechselbeziehung zwischen Landschaft, menschlichen Handlungen und kulturellen Mustern im Mittelpunkt steht, konzentriert sich die Untersuchung der Siedlungsmerkmale auf chaîne opératoire und Analysen der räumlichen Verteilung, um Aktivitätsbereiche zu erkennen. Die Untersuchung der Bestattungen nutzt Methoden der Sozial- und Kognitiven Archäologie, um sinnvolle soziale Praktiken zu rekonstruieren (Abb. 8). ${ }^{24}$

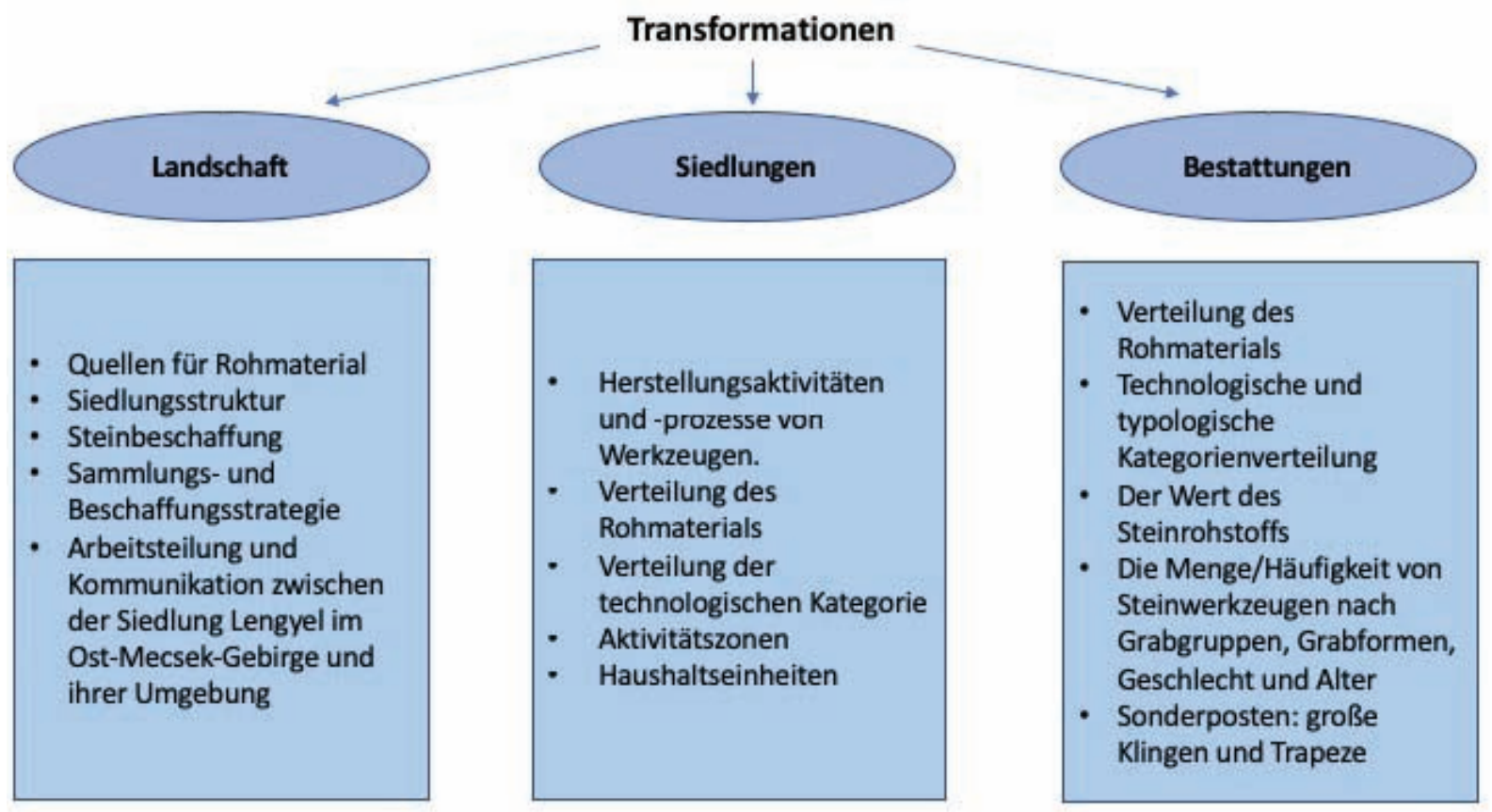

$A b b$. 8. Die Themen der Dissertation zusammenfassen.

\section{Erzielte wissenschaftliche Ergebnisse}

In den letzten Jahren habe ich das gesamte spätneolithische lithische Material der Fundstelle Alsónyék bearbeitet. Die grundlegenden Merkmale des Silexmaterials wurden im Hinblick auf die oben besprochenen Forschungsthemen analysiert. Vor diesem Hintergrund sind die wichtigsten Ergebnisse:

- 1. Wir verstehen nun die typische Rohstoffbeschaffungsstrategie, ihre Methoden und die möglichen Handels- bzw. Austauschwege, die von den spätneolithischen Gemeinschaften von Alsónyék und der gesamten südöstlich-transdanubischen Gruppe der Lengyel-Kultur genutzt wurden.

23 Zalai-GaÁl 1980; Zalai-GaÁl 1982; BÁnffy 1991; Zalai-GaÁl 1993; BÁnfFy 1994; BÁNFfy 1995; BÁNFFY 2007; ZALAI-GAÁL 2008.

24 MÜLLER 2018. 
- 2. Wir können die Tätigkeit der Rohstoffauswahl, das technologischen Wissen der Gemeinde in Alsónyék und die Organisation der Werkzeugproduktion innerhalb der Siedlung besser verstehen.

- 3. Ein besseres Verständnis vieler neuer Elemente der Bestattungspraxis, ausgehend von dem grundlegenden Punkt der in den Gräbern deponierten Steinwerkzeuge. Das Rohmaterial, die Werkzeugtypen und ihre Position im Inneren des Grabes geben spezifische Bedeutungen an. Diese Merkmale weisen auf die grundlegende Veränderung hin, die sich am Übergang vom Spätneolithikum zur frühen Kupferzeit vollzieht, und sind Teil davon.

Die Rekonstruktion der Methoden der Rohstoffbeschaffung bringt auch Erkenntnisse über die Umweltnutzung im Zusammenhang der Lengyel-Kultur. Die ursprüngliche Frage lautete: Wie wurde die Rohstoffbeschaffung organisiert und gab es eine Zusammenarbeit oder möglicherweise eine Arbeitsteilung zwischen den zeitgenössischen Siedlungen? Auf der Grundlage des Feldsurveys und des Studiums der verarbeiteten lithischen Inventare können wir schließen, dass die lokale Rohstoffbeschaffungstätigkeit keine sehr elaborierten oder spezialisierten Aktivitäten erforderte, sondern lediglich sehr gute Rohstoffkenntnisse. Wir können davon ausgehen, dass die an diesen Aktivitäten beteiligten Personen die Rohstoffquellen im OstMecsek-Gebirge sehr gut kannten. Bei der Felduntersuchung stellten wir fest, dass der größere Teil des Tals und des Baches potenzielle Rohstoffquellen waren, aus denen der Silex-Spezialist die qualitativ hochwertigen, nutzbaren Rohstoffe auf sehr einfache Weise auswählen und sammeln konnte. Was jedoch das lithische Material aus Alsónyék betrifft, so mussten die als Grabbeigaben verwendeten großformatigen Radiolaritklingen aus Aufschlüssen im Gebirge gewonnen werden, da entsprechend große Radiolaritblöcke nicht aus dem Bachtal geborgen werden konnten. Diese Gesteine sind potentiell geeignete Rohstoffquellen für diese Klingen, und in diesen dünnschichtigen Strukturen befinden sich viele Radiolariteinlagerungen. ${ }^{25}$ Diese Beobachtung deutet darauf hin, dass auch hier die im Gestein eingebetteten (Rohmaterial)-Blöcke ohne besondere Ausbeutungsaktivitäten oder spezielle Werkzeuge gesammelt werden konnten. Die größeren Radiolaritblöcke - so die Schlussfolgerung - waren geeignet, um Kerne zu erzeugen, die für die großformatigen Klingen benötigt wurden. All dies deutet darauf hin, dass die Rohmaterialsammeltätigkeit höchstwahrscheinlich von denjenigen Personen durchgeführt wurde, die über die Fähigkeiten zur Herstellung von Steinwerkzeugen verfügten, das lokale Umfeld und die genauen Orte des Flusstals und die Aufschlüsse mit geeignetem Rohmaterial für die großen Klingen kannten (Abb. 9).

In den Siedlungsinventaren gibt es keine großen Mengen an Dekortikationsabschlägen, die eine Präparation von Kernen innerhalb der Siedlung belegen würden. Aus diesem Grund können wir davon ausgehen, dass das lokale Rohmaterial in Form von Kernen, Vorkernen oder Rohmaterialblöcken in die Siedlung gelangt ist, alles Formen, die keine signifikante Menge an Kortex aufweisen. Dafür gibt es zwei mögliche Erklärungen. Eine Möglichkeit ist, dass in einigen Fällen viele Steinwerkzeuge aus dem weniger „reinen“ Radiolarit oder Teile des Ganzen verkieselt waren, so dass es nicht notwendig war, den gesamten Kortex zu entfernen. Die andere mögliche Erklärung für das Fehlen von Kortex in der Siedlung ist, basierend auf der Beobachtung des Surveys, dass es notwendig war, die Knollen und die größeren Rohmaterial- 

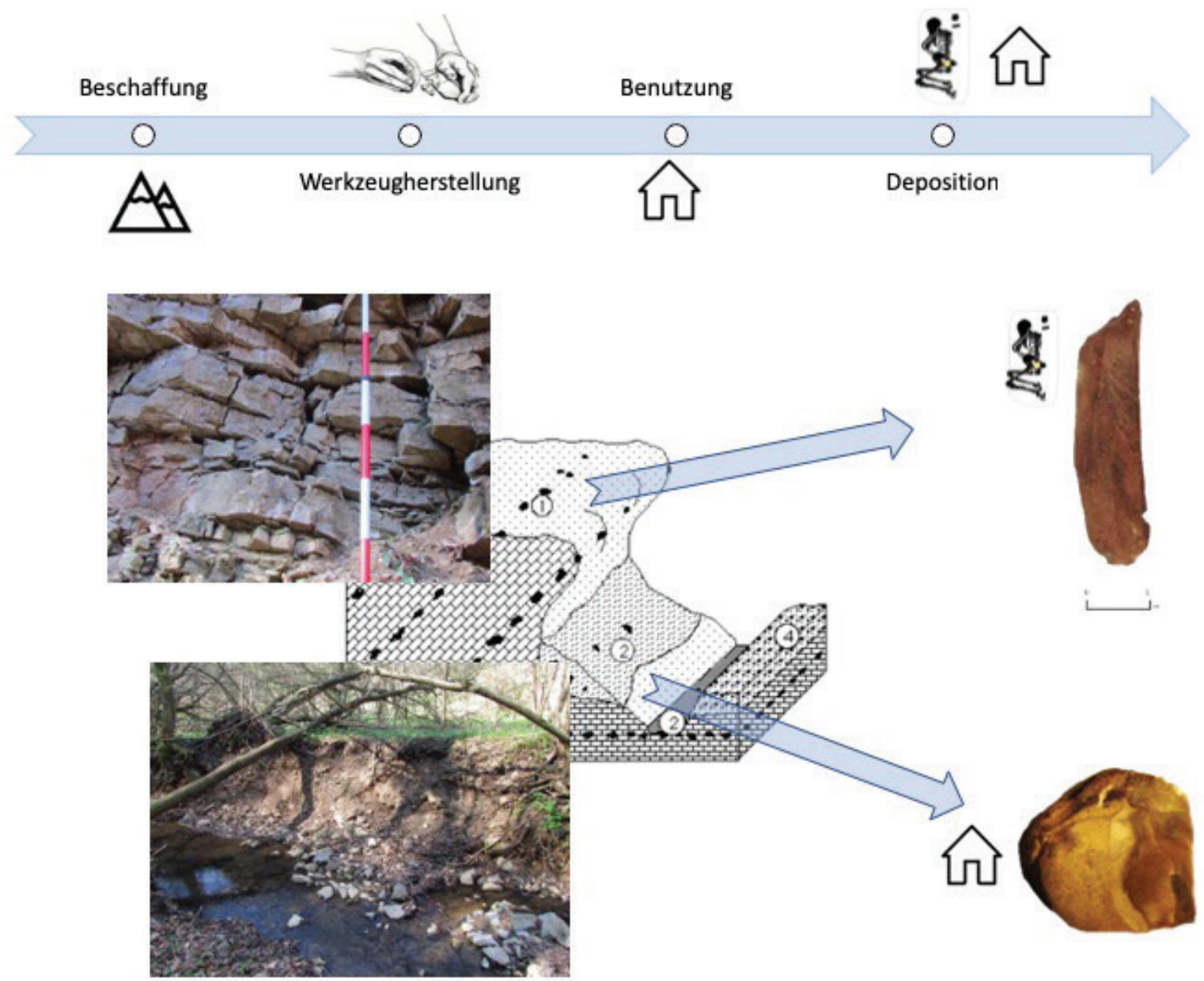

$A b b$. 9. Die Quellen der verschiedenen Arten von Rohmaterial. Die zwei möglichen Strategien der Rohmaterialbeschaffung: 1 - primäre autochthone Quelle - Bettgestelle - große Klinge aus der Bestattung, 2 - sekundäre autochthone Quelle - Bachbett - mittelgroßer Kern aus der Siedlung. Erstellt von Kata Szilágyi.

blöcke aufzubrechen, um zu prüfen, ob sie für die weitere Bearbeitung geeignet sind. Dies führt häufig zur Bildung sehr scharfer Bruchflächen und -kanten, die für den Sammler ein Problem darstellen würden, da sie leicht Verletzungen beim Transport verursachen oder einfach durch den Behälter schneiden könnten, in dem sie gelagert wurden (z.B. einen Lederbeutel). Dies stellt einen wichtigen praktischen Aspekt im Spätneolithikum dar, wenn man die Bedingungen für den Transport des Rohmaterials entweder durch lebende Tiere oder durch den Menschen berücksichtigt. Im Fall von Alsónyék würde dies unter Berücksichtigung der Ergebnisse des Surveys im Mecsek-Gebirge einen Transport über eine Entfernung von 20-30 km bedeuten. Somit wäre der Transport von unverarbeitetem oder frisch gebrochenem Gestein, dessen Rohstoff zu einem erheblichen Teil für die Herstellung von Steinwerkzeugen unbrauchbar wäre, eine zu große Energieverschwendung. Es wird wohl daher als rationeller angesehen worden sein, die Vorform oder sogar die Kerne in der Umgebung des Sammelgebietes herzustellen.

Die Untersuchung der verschiedenen technologischen Kategorien der Steinwerkzeugproduktion ermöglicht es uns, die kulturellen Traditionen der Lengyel-Kultur, die sich in den Steinwerkzeugen widerspiegelt, zu beurteilen. Aufgrund der untersuchten technologischen Merkmale war die südöstlich-transdanubische Gruppe der Lengyel-Kultur eine „Klingenkultur“, 
da in jeder lithischen Assemblage Klingen und auf Klingen gefertigte Werkzeuge stark dominieren. Den höchsten Anteil an Klingen findet man auf dem Teil der lithischen Assemblage, der aus den Gräbern stammt. Die meisten Bestattungen sind an den Fundorten Alsónyék und Mórágy zu finden, und die Anzahl der Klingen ist in den beiden Inventaren dieser Orte außerordentlich hoch. Im Allgemeinen sind in den lithischen Inventaren der Siedlungen (Zengővárkony, Pécsvárad, Villánykövesd, Alsónyék) Klingen und Abschläge gleich häufig, während Kerne und Werkzeuge seltener vorkommen. ${ }^{26}$ Im Gegensatz dazu kommen in den Bestattungskontexten Klingenwerkzeuge am häufigsten vor. Die neuen Daten aus Alsónyék können ein neues Licht auf den Grund werfen, warum die Klinge der wichtigste Typ war.

An keinem anderen Ort innerhalb der südosttransdanubischen Gruppe der Lengyel-Kultur wurden Bestattungsaktivitäten von so hoher Intensität wie in Alsónyék, oder ähnliche Mengen von Silexinventaren gefunden. Dieses Phänomen, das gleichzeitige Auftreten hochintensiver Bestattungsaktivitäten und großer Mengen an geschlagenem Steinmaterial, wirft an sich schon ein neues Licht auf die erwähnte Gruppe und die Lengyel-Kultur. Die überwiegende Mehrheit der 2236 identifizierten Bestattungen an dieser Fundstelle wies eine übliche, traditionelle ovale Grabgrube auf. Darüber hinaus wurden aber auch von den ausgrabenden Archäologen insgesamt 130 rechteckige Bestattungen dokumentiert, die bisher eine unbekannte Grabform in der Lengyel-Kultur darstellten. ${ }^{27}$ Die Verteilung der Bestattungen und Grabgruppen zeigt einen großen Unterschied in der Gesamtanlage, denn ebenso wie die Intensität der Siedlungsstruktur ist auch die Bestattungsaktivität im nördlichen Teil der Anlage am dichtesten, wo 41 von insgesamt 92 Grabgruppen in diesem Gebiet liegen. ${ }^{28}$ Die Grabgruppen weisen auch große Unterschiede in der Anzahl der Bestattungen auf, was sich in mehreren Aspekten manifestiert (Abb. 10).

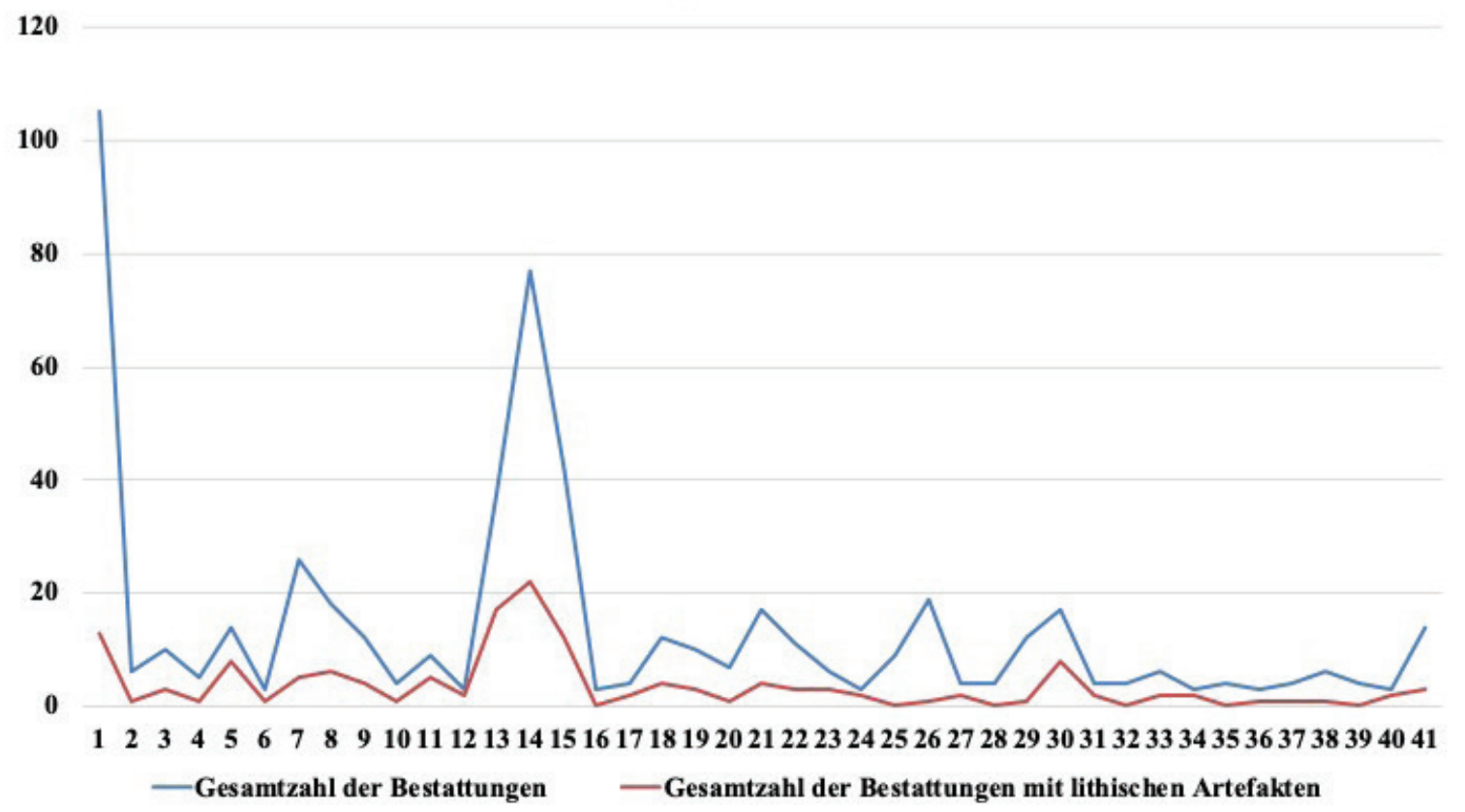

Abb. 10. Der Vergleich der gesamten Bestattungsnummer und Bestattungen mit lithischen Artefakten im Falle von 41 Grabgruppen.

26 T. BIRÓ 1998.

27 ZALAi-GAÁL et al. 2011; ZALAI-GAÁL et al. 2012a.

28 GAllina 2009; GALlina et al. 2010; KÖHLER 2013. 
Die Bildung von Grabgruppen ist Teil eines Prozesses mentaler Transformation, der Entstehung und Festigung eines neuen Bestattungsrituals. Dieses umfasst die Artefakte und Personen (in diesem Fall die Toten/Begrabenen), eine Gemeinschaft der Toten, die Bestattung als sozialen Akt, eingebettet in einen regelhaften rituellen Prozess,. Sie stellt auch eine sozio-mentale Auseinandersetzung dar, in deren Folge sich der neue Begriff des Friedhofs (die Gemeinschaft der Toten) als Bereich getrennt von der Siedlung (die Gemeinschaft der Lebenden) entwickelt. Die räumliche Trennung ist ein sehr wichtiges Element in dieser sozio-rituellen Transformation, da die aufgelisteten Gegenstände physisch voneinander abgesetzt sind. Die Suche nach und Entscheidung für einen Ort wurde in der mentalen Karte der Gemeinschaft durchgeführt, die sich erst herauskristallisieren musste, bevor die physische Anlage der Gräber und der Deponierung der Artefakte durchgeführt werden konnte. Meiner Meinung nach können wir im Fall von Alsónyék die in Grabgruppen organisierten Bestattungen und die konstanten Orte der Artefakte/Grabbeigaben im Inneren des Grabes als den nonverbalen Ausdruck dieser mentalen Karte interpretieren. ${ }^{29}$

Die Grabgruppen zeigen sehr große Unterschiede in der Anzahl der Bestattungen, ihrer Lage, Struktur, der Form der Gräber und der Häufigkeit der Steinwerkzeuge als Beigaben. Die Anzahl der Bestattungen in jeder Grabgruppe bestimmte jedoch nicht die Häufigkeit der ihnen beigegebenen Silexartefakte, tatsächlich stammte fast die Hälfte lithischen Artefakte aus Bestattungen, die keiner Grabgruppe angehörten. Die Ausrichtung und die Lage der Körperhaltung der Verstorbenen zeigen eine gewisse Regelmäßigkeit, d.h. es ist eine Art System erkennbar. Demgegenüber gibt es eine Reihe von ,Unregelmäßigkeiten“ in der Gruppenstruktur, sowohl in der Quantität als auch in der Qualität der Bestattungen und Beigaben. Vermutlich manifestieren sich Regelmäßigkeit und die Regel selbst zu dieser Zeit im Raum, d.h. der definitive Ort der Gräber und die Positionen der Grabbeigaben innerhalb des Grabes sprechen für den laufenden sozio-mentalen Wandel, der sich in dieser Zeit und in dieser spätneolithischen Gemeinschaft entfaltet. Innerhalb einer solchen Transformation lassen sich die genannten „Unregelmäßigkeiten“ gut als Abweichungen von dem sich langsam herauskristallisierenden neuen Standard erklären, in dem der sich bildende Bestattungsritus bezüglich der Individuen und der Artefakte erst allmählich seinen Platz gefunden hätte. Die Beziehung zwischen jeder individuellen Bestattung und den spezifischen Artefakten, die ihr beigegeben werden, beginnt nun aber eine bestimmte und regelmäßige Form anzunehmen. So kann sich die Beziehung zwischen dem verstorbenen Individuum oder der Bestattungsgemeinschaft und den im Grab befindlichen Artefakten während dieser Zeit entwickeln und zu einem definitiveren Zeichen werden.

\section{Literaturhinweise}

ANDREFSKY, W. J. R. 2008: Lithic technology: measures of production, use, and curation. Cambridge.

Andrefsky, W. J. R. 2009: The Analysis of Stone Tool Procurement, Production, and Maintenance. Journal of Archaeological Research 17, 65-103.

BARABÁs, A. 1986: Upper Jurassic-Lower cretaceous Radiolaria from the Mecsek Mts. In: T. BIRó, K. (szerk.): Őskori kovabányászat és kőeszköznyersanyag-azonositás a Kárpát-medencében 1. Budapest, 131-140. 
BÁCSKAY, E. 1989: A lengyeli kultúra néhány DK-dunántúli lelőhelyének pattintott kőeszközei (Chipped stone implements in some localities of Lengyel Culture in SE-Transdanubia). Communicationes Archaeologicae Hungariae, 5-21.

BÁcsKAY, E. 1990: A lengyeli kultúra pattintott kőeszközei a DK-Dunántúlon II. (The chipped stone industry of Lengyel culture in South-East Transdanubia II.) Communicationes Archaeologicae Hungariae, 59-66.

BÁcskay, E. - T. Bıró, K. 1984: A lengyeli lelőhely pattintott kőeszközeiről. A szekszárdi Béri Balogh Ádám Múzeum Évkönyve 12 (1984), 43-67.

BÁNFFy, E. 1991: Continuity or discontinuity? Some questions on the transition from the Neolithic to the Copper Age in the Carpathian Basin. Antaeus 19-20, 23-33.

BÁnffy, E. 1994: Transdanubia and Eastern Hungary in the Early Copper Age. A nyíregyházi fósa András Múzeum Évkönyve 36 1994, 291-294.

BÁNFFy, E. 1995: Über den Ausklug der Lengyel-Kultur in Transdanubia. In: KovÁcs, T. (Hrsg.): Neuere Daten zur Siedlungsgeschichte und Chronologie der Kupferzeit des Karpatenbeckens. Inventaria Praehistorica Hungariae 7. Budapest, 11-28.

BÁnfFy, E. 2007: Neolithic and Copper Age settelment patterns in the Little Balaton region and the Balaton Uplands. In: ZATyкó, Cs.-JuhÁsz, I.-SÜMEgI, P. (eds): Environmental archaeology in Transdanubia. Varia Archaeologica Hungarica 20, 97-105.

BÁnffy, E. - Osztás, A. - Oross, K. - Zalai-GaÁl, I. - Marton, T. - Nyerges, É. Á. - Köhler, K. Bayliss, A. - Hamilton, D. - Whittle, A. 2013: The Alsónyék story: towards the history of a persistent place. Bericht der Römisch-Germanischen Kommission 94, 283-318.

Bánffy, E. - Zalai-GaÁl, I. - Marton, T. - Oross, K. - Osztás, A. - Petrasch, J. 2014: Das Sárköz im südungarischesn Donaugebiet - ein Korridor zwisches dem Balkan und Mitteleuropa im 6-5. Jt. v. Chr. In: Schier, W. - Draşovean, F. (eds): The Neolithic and Eneolithic in Southeast Europe. New Approaches to Dating and Cultural Dynamics in the $6^{\text {th }}$ to $4^{\text {th }}$ Millenium BC. Prähistorische Archäologie in Südosteuropa 28. Rahden-Westfalen, 327-368.

T. BıRó, K. 1984: Őskőkori és kőkori pattintott kőeszközeink nyersanyagának forrásai (Sources of lithic raw materials for chipped implements in Hungary). Archaeologia Értesitó 111, 42-52.

T. BIRó, K. 1988: Distribution of lithic raw materials on prehistoric sites. Acta Archaeologica Academiae Scientiarum Hungaricae 40, 251-274.

T. BIRó, K. 1989: A lengyeli kultúra dél-dunántúli kőeszköz-leletanyagainak nyersanyagáról (On the Raw Material of the Chipped Stone Industry of South-Transdanubian Lengyel-Culture sites I). Communicationes Archaeologicae Hungariae, 22-31.

T. BıRó, K. 1990: A lengyeli kultúra dél-dunántúli kőeszköz-leletanyagainak nyersanyagáról 2. (On the raw material of the chipped stone industry of South-Transdanubian Lengyel culture sites. Part II.) Communicationes Archaeologicae Hungariae, 66-76.

T. BIRó, K. 1998: Lithic implements and the circulation of the raw materials in the Great Hungarian Plain during the Late Neolithic Period. Budapest.

Dombay, J. 1939: A zengővárkonyi őskori telep és temető. Archaeologia Hungarica 23. Budapest.

Dombay, J. 1958: Kőrézkori és kora vaskori település nyomai a Pécsváradi Aranyhegyen (Überreste einer aeneolithischen und früheisenzeitlichen Ansiedlung an Berg Arany (Goldberg) bei Pécsvárad). A Janus Pannonius Múzeum Évkönyve, 53-102.

Dombay, J. 1960: Die Siedlung und das Gräberfeld in Zengővárkony. Archaeologia Hungarica 37. Budapest - Bonn.

Dzbyński, A. 2008: Ritual and Understanding. Rational Bases of Communication and Exchange in Prehistoric Europe. Rzeszów.

Gallina, Zs. 2010: Bátaszék, M6 TO-11 lelőhely. In: Kisfaludi, J. (szerk.): Régészeti Kutatások Magyarországon 2009 (Archaeological Investigations in Hungary 2009). Budapest, 144. 
Gallina, Zs. - Hornok, P. - Paluch, T. - Somogyi, K. 2010: Előzetes jelentés az M6 AP TO 10/B és 11. számú lelőhelyrészen végzett megelőző feltárásról. Alsónyék-Bátaszék (Tolna megye) 20062009 (Vorbericht über die präventive Ausgrabung am Fundortsteil Nr. M6 AP To 10/B und 11. Alsónyék-Bátaszék (Komitat Tolna) 2006-2009). AWosinsky Mór Múzeum Évkönyve 32, 7-100.

Holló, Zs. - Lengyel, Gy. - Mester, Zs. 2001: Egy pattintott kőeszköz életútja. Magyar kifejezések a technológiai vizsgálatokhoz 1. Ósrégészeti Levelek 3, 51-57.

Holló Zs. - Lengyel Gy. - Mester, Zs. 2002: Egy pattintott kőeszköz elkészítése: rendszer és technika. Magyar kifejezések a technológiai vizsgálatokhoz 2. Ősrégészeti Levelek 4, 98-104.

Holló, Zs. - Lengyel, Gy. - Mester Zs. - SzolyÁK, P. 2004: Egy pattintott kőeszköz vizsgálata. Magyar kifejezések a technológiai vizsgálatokhoz 3. Ösrégészeti Levelek 6, 62-80.

Inizan, M. L. - Reduron-Ballinger, M. - Roche, H. - Tixier, J. 1999: Technology and Terminology of Knapped Stone. Nanterre, 1999.

KondA, J. 1986: The Mezozoic siliceous rocks of the Transdanubian Mid-Mountains. In: T. Biró, K. (szerk.): Őskori kovabányászat és kőeszköznyersanyag-azonositás a Kárpát-medencében. 1. kötet. Budapest, 165-168.

KöHLER K. 2012: A késő neolitikus Lengyeli kultúra népességének biológiai rekonstrukciója. PhD dissertation, Budapest.

KöHLER, K. 2013: Biological reconstruction of the Late Neolithic Lengyel Culture. Dissertationes Archaeologicae 3/1, 179-204.

MÜLLER, J. 2018: The Disentanglement of Landscapes. Remarks on Concepts of Socio-Environmenthal Research and Landscape Archaeology. In: HAUg, A. - Käppel, L. - Müller, J. (eds): Past Landscapes. The Dynamics of Interaction between Society, Landscape and Culture. Leiden, 39-52.

Odell, G. H. 2006: Lithic Analysis. University of Tulsa, Oklahoma.

Osztás, A. - ZALAI-GAÁL, I. - BÁNFFy, E. 2012: Alsónyék-Bátaszék: a new chapter in the research of the Lengyel culture. Documenta Praehistorica 39, 377-396.

Osztás, A. - BÁnffy, E. - ZAlai-GaÁl, I. - Oross, K. - Marton, T. - Somogyi, K. 2013a: AlsónyékBátaszék: introduction to a major Neolithic settlement complex in south-east Transdanubia, Hungary. Bericht der Römisch-Germanischen Kommission 94, 7-22.

Osztás, A. - Zalai-GaÁl, I. - BÁnffy, E. - Marton, T. - Nyerges, É. Á. - Köhler, K. - Somogyi, K. Gallina, Zs. - Bronk Ramsey, C. - Dunbar, E. - Kromer, B. - Bayliss, A. - Hamilton, D. Whittle, A. 2013b: Coalescent community at Alsónyék: the timings and duration of Lengyel burials and settlement. Bericht der Römisch-Germanischen Kommission 94, 180-361.

RACzky, P. 1974: A lengyeli kultúra legkésőbbi szakaszának leletei a Dunántúlon 8 The finds of the latest phase of the Lengyel Culture in Transdanubia). Archaeologiai Értesitó 101, 185-210.

RACZKY, P. 1998: Kulturális kapcsolatok az Alföld és a Dunántúl között a késői neolitikum időszakában. In: Asztalos I. - AszTalos T. (szerk.): Egy múzeum szolgálatában. Negyven éves az aszódi Petőfi Múzeum. Tanulmányok Asztalos István tiszteletére. Szentendre - Aszód, 9-19.

RACZKY, P. 2002: Evidence of contacts between the Lengyel and Tisza-Herpály cultures at the late neolithic site of Polgár-Csőszhalom. Budapest Régiségei 36, 79-92.

RACzky P. - ANDERs A. 2009: Tér és időszemlélet az újkőkorban. Polgár-Csőszhalom ásatási megfigyelései. In: Anders A. - Szabó M. - Raczky P. (szerk.): Régészeti dimenziók. Tanulmányok az ELTE BTK Régészettudományi Intézet tudományos műhelyéből. Budapest, 75-92.

RACZKy, P. - Anders, A. 2014: Acitivity loci and data for spatial division at a Late Neolithic site-complex (Polgár-Csőszhalom: a case study). In: Hansen, S. (Hrsg.): Leben auf dem Tell als soziale Praxis. Beiträge des internationalen Symposiums in Berlin vom 26.-27. Februar 2007. Kolloquien zur Vor- und Frühgeschichte 14. Bonn, 143-163.

RACZKy, P. - Anders, A. - Siklósi, Zs. 2014: Trajectories of continuity and change between the Late Neolithic and The Copper Age in Eastern Hungary. In: Schier, W. - Draşovean, F. (eds): 
The Neolithic and Eneolithic in Southeast Europe. New Approaches to dating and cultural dynamics in the 6th to 4th millennium BC. Prähistorische Archäologie in Südosteuropa 28. Rahden/ Westf., 319-346.

Raucsik B. 2012a: Fonyászói Mészkő Formáció. In: Fózy I. (szerk.): Magyarország litosztratigráfiai alapegységei. Fura. Budapest, 180-183.

Raucsik B. 2012b: Komlói Mészmárga Formáció. In: Fózy I. (szerk.): Magyarország litosztratigráfiai alapegységei. Jura. Budapest, 174-176.

RAucsik B. 2012c: Óbányai Mészkő Formáció. In: Fózy I. (szerk.): Magyarország litosztratigráfiai alapegységei. Jura. Budapest, 177-179.

RAucsıк B. 2012d: Mecseknádasdi Homokkő Formáció. In: Fózy I. (szerk.): Magyarország litosztratigráfiai alapegységei. Jura. Budapest, 159-163.

Regenye, J. 2000: A lengyeli kultúra újabb lelőhelyei Veszprém megyében (New sites of the Lengyel culture in Veszprém county). Veszprém Megyei Múzeumok Közleményei 21, 9-19.

Regenye, J. 2011: Kö és agyag. Település és életmód a neolitikum-rézkor fordulóján a Dunántúlon. Veszprém Megyei Múzeumok Igazgatósága, Veszprém.

SAlisbury, R. 2012: Place and identity: networks of Neolithic communities in Central Europe. Documenta Praehistorica 39, 203-213.

Schier, W. 2014: The Copper Age in Southeast Europe - historical epoch or typo-chronological construct? In: Schier, W. - Draşovean, F. (eds): The Neolithic and Eneolithic in Southeast Europe. New Approaches to Dating and Cultural Dynamics in the 6th to 4th Millenium BC. Prähistorische Archäologie in Südosteuropa 28. Rahden/Westfalen, 419-435.

Serlegi, G. - Rassmann, K. - Osztás A. - Mischia, C. - Furholt, M. - Ohlrau, R. - Winkelman, K. BÁNFFy, E. 2013: Neolitikus lelőhelyek nagyfelületű magnetométeres felmérése a Kalocsai és Tolnai Sárközben. Magyar régészet online magazin, 2013 tavasz, 1-8.

SikLósi Zs. 2010: A társadalmi egyenlőtlenség nyomai a késő neolitikumban a Kárpát-medence keleti felén. PhD Dissertation, Budapest.

SiklósI, Zs. 2013: Traces of Social Inequality during the Late Neolithic in the Eastern Carpathian Basin. Dissertation Pannonicae 4/3. Budapest.

SzILÁGyI, K. 2017a: Approaching household units from chipped stone assemblages at AlsónyékBátaszék, south Hungary. Bulgarian e-fournal of Archaeology 7, 61-83.

SZILÁgYI, K. 2017b: Preliminary result of the lithic raw material distribution at the Alsónyék-Bátaszék Late Neolithic site (Southeastern Hungary). In: ForŢıu, S. (ed.): Archeovest V1 - In honorem Doina Benea - Interdisciplinaritate în Arheologie și Istorie. Szeged, 99-129.

SzILÁGyi, K. 2018a: A Field survey of knappable raw materials in the Eastern Mecsek area. Hungarian Archaeology E-fournal, 2018 Summer, 1-9.

SzILÁgyi, K. 2018b: Lithic Raw Material Procurement in the Late Neolithic Southern-Transdanubian Region: A Case Study from the Site of Alsónyék-Bátaszék. Archaeologia Polona 56, 123-136.

ZALAI-GAÁL, I. 1980: Adatok a lengyeli kultúra kutatásának történetéhez (Data to the history of research of the Lengyel Culture). Archaeologiai Értesitó 107, 176-186.

ZALAI-GAÁL, I. 1982: A lengyeli kultúra a Dél-Dunántúlon (Die Siedlungsverhältnisse der Lengyel-Kultur in SW-Ungarn). A szekszárdi Béri Balogh Ádám Múzeum Évkönyve 10-11, 3-58.

ZALAI-GAÁL, I. 1986: Sozialarchäologische Forschungsmöglichkeiten aufgrund spätneolithischer Gräbergruppen in Südwestlichen Ungarn. A szekszárdi Béri Balogh Âdám Múzeum Évkönyve 13, 139-154.

ZALAI-GAÁL, I. 1988: Közép-európai neolitikus temetők szociálarchaeológiai elemzése (Sozialarchäologische Untersuchungen des mitteleuropäischen Neolithikums aufgrund der Gräberfeldanalyse). A szekszárdi Béri Balogh Ádám Múzeum Évkönyve 14, 9-178. 
ZALAI-GAÁL, I. 1993: A lengyeli kultúra kronológiai problémái a Dél-Dunántúlon. A Janus Pannonius Múzeum Évkönyve 37, 79-91.

ZALAI-GAÁL I. 2001: A késő neolitikum története a Dél-Dunántúlon a temetőelemzések tükrében. Tipológia - Kronológia - Társadalomrégészet. Akadémiai Doktori Értekezés. Budapest.

Zalai-GaÁl, I. 2002: Die Neolithische Gräbergruppe-B, von Mórágy-Túzkődomb. I. Die Archäologischen Funde und Befunde. Wosinsky Mór Múzeum, Szekszárd-Saarbrücken.

ZALAI-GAÁL, I. 2008: An der Wende vom Neolithikum zur Kupferzeit in Transdanubien (Ungarn): Die „Häuptlingsgräber” der Lengyel-Kultur in Alsónyék-Kanizsa. Altertum 53/4, 241-280.

ZAlAi-GaÁL, I. - GÁL, E. - KöHLER, K. - Osztás, A. 2011: Das Steingerätedepot aus dem Häuptlingsgrab 3060 der Lengyel-Kultur von Alsónyék, Südtransdanubien. In: BEIER, H.-J. - EINICKE, R. Biermann, E. (Hrsg.): Dechsel, Axt, Beil \& Co - Werkzeug, Waffe, Kultgegenstand? Aktuelles aus der Neolithforschung. Beiträge der Tagung der Arbeitsgemeinschaft Werkzeuge und Waffen im Archäologischen Zentrum Hitzacker 2010 und Aktuelles. Langenweissbach 2011. Beiträge zur Urund Frühgeschichte Mitteleuropas 63. - Varia Neolithica 7. Langenweißbach, 65-83.

ZALAI-GAÁL, I. - KöHLER, K. - OszTÁs, A. 2012a: Totenbrett oder Totenhütte? Zur Struktur der Gräber der Lengyel-Kultur mit Pfostenstellung in Südtransdanubien. Acta Archaeologica Academiae Scientiarum Hungaricae 63, 69-116.

Zalai-GaÁl, I. - Köhler, K. - OsztÁs, A. - Szilágyi, K. 2012b: Präliminarien zur Sozialarchäologie des lengyelzeitlichen Gräberfeldes von Alsónyék-Bátaszék, Südtransdanubien. Prähistorische Zeitschrift 87/1, 58-82.

ZALAI-GAÁL, I. - OsZtÁs, A. 2009: A lengyeli kultúra települése és temetője Alsónyék-Kanizsa-dűlőben. In: Bende L. - LőRInczy G. (szerk.): Medinától Etéig. Régészeti Tanulmányok Csalog József születésének 100. évfordulójára. Szentes, 245-254. 
